\title{
Symbolic dynamical unfolding of spike-adding bifurcations in chaotic neuron models
}

\author{
R. Barrio ${ }^{1,2}$ (a), M. Lefranc ${ }^{3}$, M. A. Martínez ${ }^{2}$ and S. Serrano ${ }^{1,2}$ \\ 1 Departamento de Matemática Aplicada and IUMA. University of Zaragoza. E-50009. Spain. \\ 2 CODY and GME. University of Zaragoza. E-50009. Spain. \\ 3 Laboratoire de Physique des Lasers, Atomes, et Molecules, CNRS, UMR8523, e Université Sciences et Technologies, \\ 59655 Villeneuve d'Ascq, France.
}

PACS 05.45.Ac - Low-dimensional chaos

PACS $05.45 . \mathrm{Pq}$ - Numerical simulations of chaotic systems

PACS 87.19.11 - Models of single neurons and networks

\begin{abstract}
We characterize the systematic changes in the topological structure of chaotic attractors that occur as spike-adding and homoclinic bifurcations are encountered in the slow-fast dynamics of neuron models. This phenomenon is detailed in the simple Hindmarsh-Rose neuron model, where we show that the unstable periodic orbits appearing after each spike-adding bifurcation are associated with specific symbolic sequences in the canonical symbolic encoding of the dynamics of the system. This allows us to understand how these bifurcations modify the internal structure of the chaotic attractors.
\end{abstract}

The wide-range assessment of brain and behaviors is one of the pivotal challenges of this century. To understand how an incredibly sophisticated system such as the brain per se functions dynamically, it is imperative to study the dynamics of its constitutive elements - neurons. Therefore, the design of mathematical models for neurons has arisen as a trending topic in science for a few decades, since Hodgkin and Huxley developed the first model of action potentials in the neuron membrane [1]. Starting from that seminal mathematical model, a lot of variant models describing different kinds of neuron cells in numerous animals have been proposed in the literature. For instance, a reduced model [2-4] of the bursting of leech heart neuron is specified by the following three equations derived through the Hodgkin-Huxley gated variable formalism:

$$
\begin{aligned}
& C V^{\prime}=-I_{\mathrm{Na}}-I_{\mathrm{K} 2}-I_{\mathrm{L}}-I_{\mathrm{app}}-I_{\mathrm{Syn}}, \\
& \tau_{\mathrm{Na}} h_{\mathrm{Na}}^{\prime}=h_{\mathrm{Na}}^{\infty}(V)-h_{\mathrm{Na}}, \\
& \tau_{\mathrm{K} 2} m_{\mathrm{K} 2}^{\prime}=m_{\mathrm{K} 2}^{\infty}(V)-m_{\mathrm{K} 2},
\end{aligned}
$$

with the steady-state values of Boltzmann gating variables given by (see [4] for a detailed description of parameter values and their biological meaning)

\footnotetext{
(a) E-mail: rbarrio@unizar.es
}

$$
\begin{aligned}
& h_{\mathrm{Na}}^{\infty}(V)=[1+\exp (500(V+0.0325))]^{-1} \\
& m_{\mathrm{K} 2}^{\infty}(V)=\left[1+\exp \left(-83\left(V+0.018+V_{\mathrm{K} 2}^{\text {shift }}\right)\right)\right]^{-1} .
\end{aligned}
$$

As control parameters of the system are varied, its dynamics undergoes bifurcations such as classified by dynamical systems theory that match qualitative metamorphoses in terms specific to neuroscience. A broad range of non-stationary activity types can be observed, which includes regular and irregular tonic spiking, bursting and mixed-mode oscillations and combinations of them, as well as oscillatory transients toward quiescent states. In terms of dynamical system theory, these behaviors correspond to stable periodic and deterministically chaotic orbits in the phase space of the model. Figure 1 represents a twoparameter sweep of the leech model in the $\left(\tau_{K 2}, I_{\mathrm{app}}\right)$ plane, where the number of spikes per period, measured with the spike-counting method (SPC) [5], is color-coded. Banded structures correlated with zones of chaotic behavior appear clearly in this diagram. They are associated with spike-adding bifurcations, where the number of spikes is incremented by one, as indicated in the figure.

Spike-adding bifurcations are special bifurcations that are common in fast-slow systems. They lead to the appearance of extra spikes (turns) in the fast manifold region and are quite important in that they progressively 


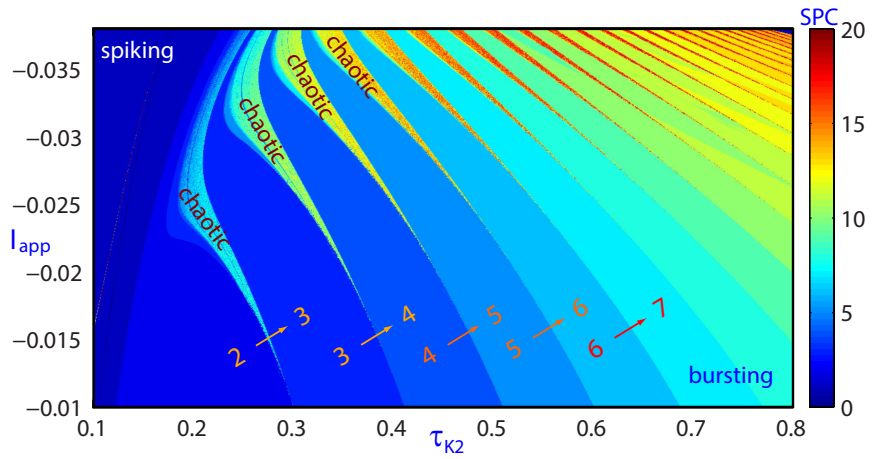

Figure 1: (Color online) $\left(\tau_{K 2}, I_{\mathrm{app}}\right)$-parametric sweep of the leech heart neuron model using the SPC approach.

modify the spectrum of periodic orbits of the system and the structure of chaotic attractors. Important examples of such fast-slow dynamics [6-8] are found in chemical reactions, laser dynamics and in mathematical neuron models. Understanding how to generate and control a burst of spikes in neuron cells, and how chaotic behavior can appear in such systems [9-11] are some of the most fundamental questions in neuroscience. The key questions that we want to address are: how is this chaotic behavior organized? How do spike-adding bifurcations influence chaotic behavior?

Most neuronal systems are strongly dissipative, with the contraction of the flow along the stable manifolds being much greater than expansion along the unstable manifolds of the equilibria [12-14]. Thus, they accept a qualitative description through one-dimensional maps, as the intersection of the attractor with a section plane can be described by a single coordinate. The standard way to obtain such maps is to compute the Poincaré First Return Map (FRM) of the attracting invariant sets or to follow data from limit cycles of the fast subsystem, like in [15].

The topological structure of the $3 \mathrm{D}$ chaotic invariant set can be described in terms of its topological template [12-14], a branched two-dimensional manifold such that all periodic orbits in the invariant set can be projected to the template without changing their knot and link invariants. This topological model can be quantified by a set of integer invariants. These characteristic numbers describe the torsion of the branches (associated with the rotation of the unstable manifold of period-1 orbits), their linking as well as how branches stack onto each other as they merge. All self-linking and linking numbers characterizing the intertwining of periodic orbits can be computed from these invariants. Conversely, computing a few knot and link invariants from these orbits then suffices to determine the template uniquely $[12,14]$. Understanding how the template and the spectrum of orbits populating it change across bifurcations is a robust way to characterize how the chaotic dynamics unfolds in parameter space. For example, it was shown recently in $[16,17]$ how a global bifurcation can increase the number of branches in the topological template of dissipative systems like the Rössler model (see also $[18,19]$ ). Here, we observe in neuron models that changes in the topological structure along a series of spike-adding bifurcations is more gradual: the number of branches does not change, however the spectrum of periodic orbits is modified in a systematic way, and accordingly how the attractor is visited by chaotic orbits. We now detail these changes.

In order to help in the analysis of neuron models simulated realistically within the Hodgkin-Huxley framework [1], a common approach is to use some simplified models. In particular, the 3D Hindmarsh-Rose (HR) model [20] reproduces fairly well the basic oscillatory activities routinely observed in isolated biological cells and in neural networks. Therefore, in our detailed analysis we will consider the HR model, but a similar analysis can be applied to other neuron and fast-slow models. The HR model is described by three nonlinear ODEs:

$$
\left\{\begin{array}{l}
\dot{x}=y-a x^{3}+b x^{2}-z+I, \\
\dot{y}=c-d x^{2}-y \\
\dot{z}=\epsilon\left[s\left(x-x_{0}\right)-z\right]
\end{array}\right.
$$

where $x$ is the membrane potential, $y$ the fast and $z$ the slow gating variables for ionic current. The parameters are typically set as follows: $a=1, c=1, d=5, s=4$, $x_{0}=-1.6, \epsilon=0.01$. We will study the system for several values of the remaining parameters: $I$, the 'external applied current', and $b$. Note that decreasing the value of the small parameter $\epsilon$ increases the number of spikes but not change the global behavior. This model fulfills the two basic conditions of being computationally simple but still able to reproduce the main behaviors (the rich firing patterns) exhibited by the real biological neurons.

In Fig. 2 we show in the main panel the $(b, I)$ parametric sweep using the SPC method. This technique permits to detect automatically the spike-adding bifurcations as the loci of parameter space where the number of spikes is incremented. We also observe a main chaotic region with different stripes, where each lobe is clearly connected with the spike-adding process, similarly to Fig. 1 for the leech heart neuron model. Focusing on the chaotic region, the evolution of the largest Lyapunov exponent along the white line $(I=(1-0.265 \cdot b) / 0.0691)$ is shown in the top panel. Color points indicate the chaotic regimes that have been selected in different lobes for further study. The chaotic attractors observed in these regimes and their FRM are shown on the left pictures. Fig. 3 depicts schematically the global organization of the chaotic region as being "onion-like" [21], with different lobes that are concentric and going deeper moving from right to left but in a exponentially small scale from left to right beginning on the other side. This structure governs directly the systematic evolution in the spectrum of UPOs and the topological structure of the chaotic attractors in squarewave bursters (or fold/hom bursters) when control param- 

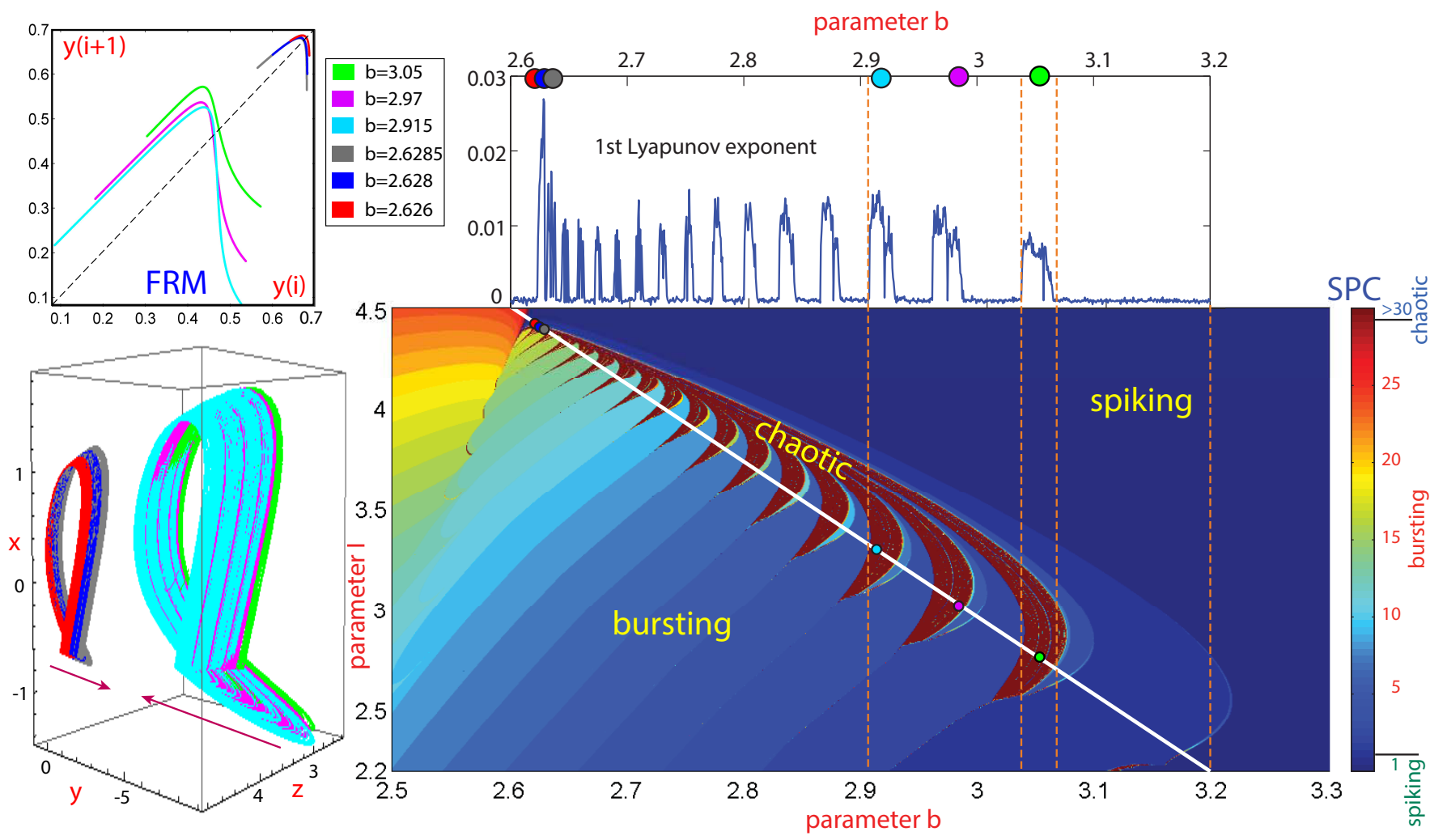

Figure 2: (Color online) (b,I)-parametric sweep of the Hindmarsh-Rose model using the SPC approach. The spike number is color-coded as indicated by the bar on the right. On top we show the variation of the Lyapunov exponent along the selected white line $(I=(1-0.265 \cdot b) / 0.0691)$. At different values of the $b$ parameter, indicated by color dots along the white line, we found different chaotic attractors shown on the left, together with their FRM which are all unimodal.

eters change. A similar structure is observed in the leech model.

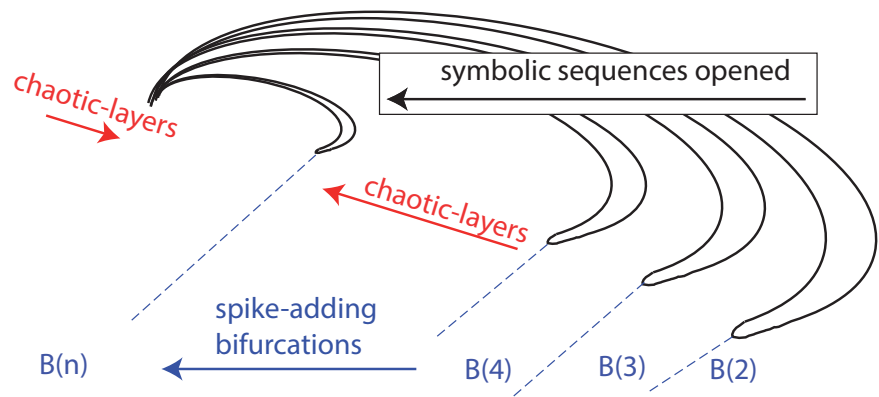

Figure 3: (Color online) Scheme of the "onion-like" structure of the main chaotic region. The chaotic layers are accumulated in the left side. The spike-adding process changes the stable periodic orbits outside the chaotic region.

Bifurcation analysis provides several insights into the spike-adding process and the creation of chaotic lobes. Fig. 4 provides a magnification of Fig. 2, specifying several codimension-one bifurcation lines: homoclinic, period-doubling, and fold bifurcations (or saddle-node of limit cycles) as well as spike-adding and some codimen- sion two orbit-flip homoclinic bifurcation points. These bifurcation lines have been obtained using the continuation software AUTO and the SPC technique. In [5,21,22], the role of these bifurcations in the spike-adding process and their influence in the creation of the chaotic structures has been shown.

Once we have located the chaotic structures and established their relation with the spike-adding process, we want to characterize their influence on the structure of the different chaotic attractors. To this aim, the appropriate tool is the study of the topological templates obtained by characterizing the intertwining of the UPOs embedded in the chaotic attractors. As illustrated in Fig. 5, a topological template is a branched surface which combines orientable and non-orientable, twisted, stripes (like a Möbius band) $[12,14]$. The procedure followed is similar to that described in previous works $[12,14,23,24]$ so that we only sketch it briefly here.

First, the lowest-period periodic orbits must be located. We typically considered orbits up to topological period 5 , where the topological period corresponds to the number of intersections with the Poincare section (which is also the number of loops of the orbit in phase space). In dynamical systems defined by a set of differential equations, this task can easily be carried out with high precision, as it is 


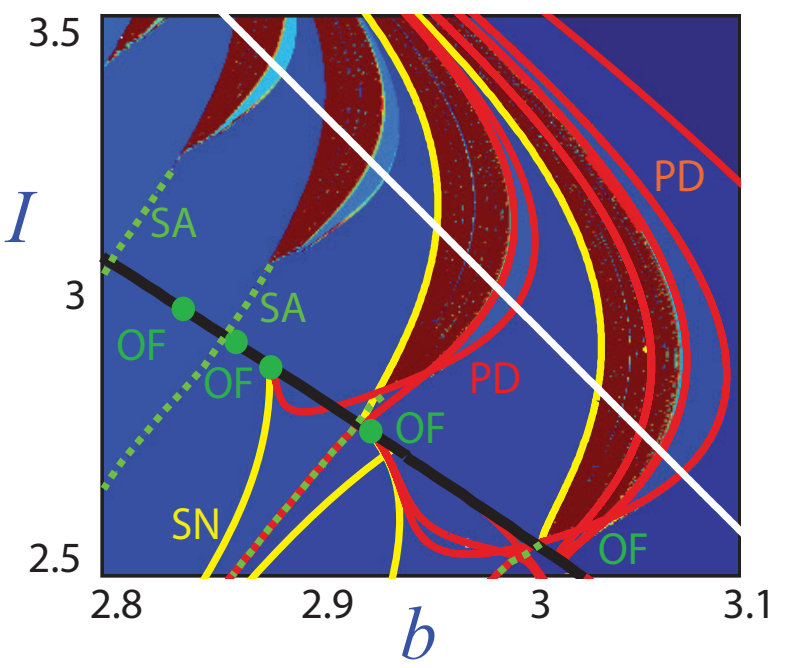

Figure 4: (Color online) Magnification of Fig. 2 with several bifurcation lines of limit cycles (Black: homoclinic bifurcation, Red: period-doubling (PD), Yellow: fold bifurcation (SN) and Green: spike-adding (SA)) and some codimension two bifurcation points (Green dots: orbit-flip (OF)).

equivalent to a zero-finding problem. Then, the intertwining of closed trajectories associated with these periodic orbits are characterized using topological invariants. Here we use topological periods and linking numbers. The linking number of a pair of orbits indicates how many turns one orbit winds around the other. In this work, we determine its value using a recently proposed algorithm [25] for computing the Gauss linking integral $[24,26]$, which is well suited to systems of differential equations (an alternative and more common method is to compute the sum of signed crossings between the projections of the two orbits onto a plane [23]).

The next step is to determine the simplest template which carries a set of periodic orbits with the same linking numbers as the detected periodic orbits. The template is easier to determine when a one-dimensional FRM is available. In our case, the FRM of the HR system is unimodal, which indicates that we only need two symbols to describe the whole spectrum of orbits and that accordingly the template should have two branches (the leech heart neuron model also presents a unimodal FRM [4]). The FRM can be used to assign a symbolic name to periodic orbits, which describes how the two branches of the FRM are successively visited, with symbol "0" (resp., "1") denoting the positive (resp., negative) slope branch. Similarly, periodic orbits on a template can be assigned a symbolic name by labeling template branches with different symbols. By definition of the template, any set of periodic orbits with given symbolic names in the attractor should have exactly the same topological invariants as periodic orbits with the same names on the template.

Given a set of periodic orbits with given symbolic names
Table 1: Number of UPOs of topological period $m$ for a few selected chaotic attractors and the symbolic forbidden chains (FCh).

\begin{tabular}{ccccccr}
$b$ & $m=1$ & $m=2$ & $m=3$ & $m=4$ & $m=5$ & FCh \\
\hline 3.05 & 1 & 1 & 0 & 1 & 2 & 00 \\
2.97 & 1 & 1 & 2 & 1 & 4 & 000 \\
2.915 & 1 & 1 & 2 & 3 & 4 & 0000 \\
\hline 2.6285 & 1 & 1 & 2 & 3 & 4 & 0000 \\
2.628 & 1 & 1 & 2 & 1 & 4 & 000 \\
2.626 & 1 & 1 & 0 & 1 & 2 & 00
\end{tabular}

and linking numbers, the equations expressing the linking number as a function of the template characteristic numbers can then be inverted to determine the template structure (see, e.g., [27] or appendix A of [14]). The template can also be determined without using a symbolic encoding. In this case, the algorithm performs a combinatorial search over all possible symbolic names to identify the set of symbolic names for which topological invariants of template orbits match those determined experimentally $[14,28,29]$. The analysis thus yields the candidate symbolic names for the periodic orbits detected as a byproduct of template determination. In our study, we followed this encoding-free approach and found that the symbolic names obtained were identical to those derived using the FRM. The symbolic dynamical analysis carried out below is therefore both dynamically and topologically significant.

In Table 1 we show the number of UPOs of different topological period for different values of the parameter $b$ along the selected line in parameter space. We also indicate the irreducible forbidden symbol chains that specify the spectrum of periodic orbits. More precisely, those are the symbol chains that never appear in the symbolic names determined from template analysis and do not contain any shorter forbidden chain. For $b=2.97$, for example, symbolic names never contain the sequence "000" but some contain "00". The list of irreducible forbidden chains defines the "grammar" of chaos.

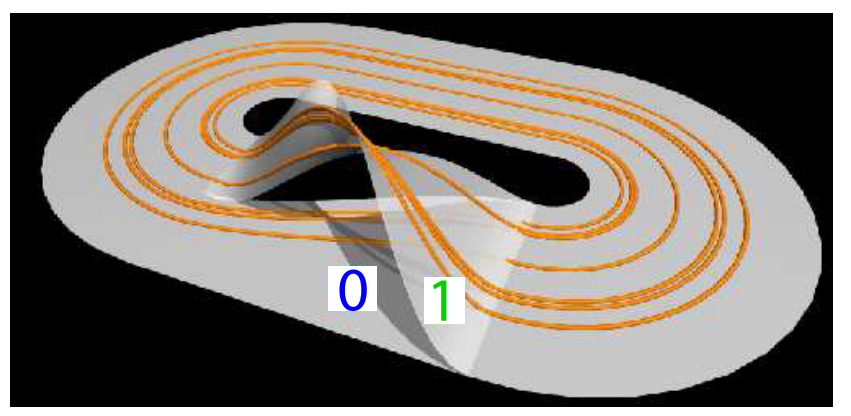

Figure 5: (Color online) Generic topological template of the Hindmarsh-Rose system (Smale template) for the complete parametric plane of Fig. 2.

Along the parameter path considered, we observe a sys- 
tematic evolution of the number of UPOs, which increases as one goes from both extremes of the path towards the center. This reinforces the "onion-like" picture of the structure of the HR bifurcation diagram (also common in other neuron systems in parameter regions with squaredwave bursters). For each parameter set, we identified lowperiod UPOs embedded in the attractor and determined the template describing their topological organization as described above. In each case, enough orbits were used to ensure that their linking numbers significantly overdetermine the template.

A remarkable result is that for all the attractors of the Hindmarsh-Rose model we analyzed, the topological template was embedded in the Smale horseshoe template $[12,14]$ represented in Fig. 5. To achieve a finer description of the chaotic regimes, we characterized them through the symbolic dynamics of their spectrum of periodic orbits, more precisely by their "grammar".

We found that the "onion-like" structure associated with the spike-adding bifurcations is easily described in terms of this grammar. When moving from the bottom right to the upper left of the path in Fig. 2, new periodic orbits with additional spikes are successively created, with more and more consecutive 0's becoming allowed in successive chaotic zones (see Table 1). A similar progressive unfolding of the symbolic dynamics has already been reported in a laser [30], however here we also observe a novel phenomenon where this unfolding is reversed: as one reaches the upper extreme end of the path, the symbol chains newly allowed disappear in a very short parameter range. For instance, from the value $b=3.05$ to $b=2.97$ the chain 00 is allowed, but from $b=2.628$ to $b=2.626$ it is again forbidden.

Each spike-adding bifurcation increases the maximum length of allowed chains of 0's by one, thus this length is bounded by the number of such bifurcations in parameter space. For a fixed value of $\epsilon$, this number is finite so that arbitrary long chains of 0's cannot be observed. When $\epsilon$ decreases, more and more spike adding bifurcations are found in the diagram (in fact, their number depends exponentially on $\epsilon$ ), which implies that the spectrum of orbits populating the topological template is closer and closer to that of the full hyperbolic Smale horseshoe template. When forbidden symbol chains exist, they give a Cantor structure to the subset of the topological template which carries out orbits mapped from the attractor orbits (see Fig. 6), the holes corresponding to orbits whose name contains the forbidden symbolic sequences. This structure reflects that of the attractor and how it evolves when control parameters are swept and spike-adding bifurcations are encountered.

In conclusion, we have shown that the topological templates of the chaotic attractors of the Hindmarsh-Rose model, and probably of other neuron models, are subtemplates of the Smale topological template. The spikeadding processes and the bifurcations associated to them are accompanied by a gradual change in the spectrum of

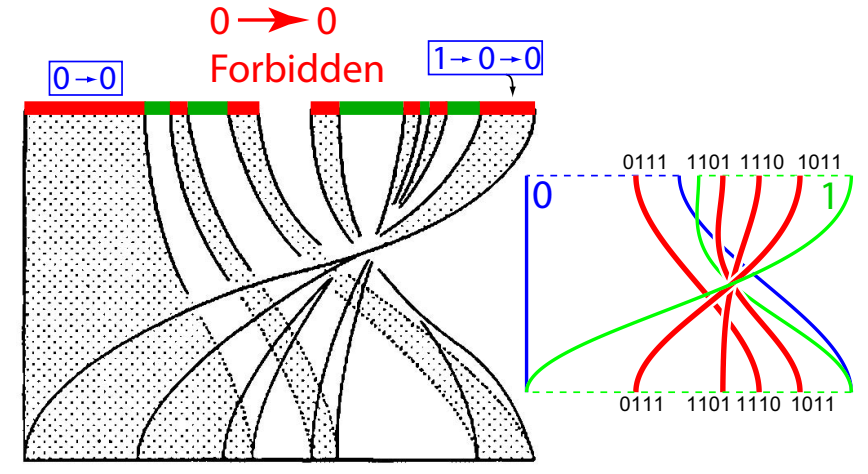

Figure 6: (Color online) Cantor-like structure of the allowed and some forbidden routes in the complete Smale topological template $(0 \rightarrow 0$ sequence forbidden $)$. On the right, example of the allowed periodic orbit 0111 in the template.

periodic orbits embedded in the attractor, and the onionlike structure in parameter space can be understood directly in terms of symbolic dynamics, each chaotic zone corresponding to a specific maximal number of consecutive 0's in the symbolic dynamics. As parameters are varied, the fine structure of the topological template and accordingly of the attractor change continuously in a way that is well described by the appearance and disappearance of symbol sequences in the symbolic dynamics. The extent to which the symbolic dynamics can approach that of the complete Smale horseshoe template (with no forbidden symbol chains) seems to be controlled by the small parameter of the fast-slow neuron model. This is consistent with the fact that according to the "pruning front conjecture" [31], less dissipative systems are characterized by more irreducible forbidden symbol sequence and thus they face more obstructions to achieve fully developed chaos. We remark that, for the Hindmarsh-Rose system, the smaller $\epsilon$, the more dissipative the system and so we are in this situation.

$$
* * *
$$

R.B. and S.S. have been supported during this research by the Spanish Research Grant MTM2012-31883 and R.B., M.A.M. and S.S. by the Diputación General de Aragón (group E48). The authors thank their colleague and friend Prof. Andrey Shilnikov for many interesting discussions and common work on this subject. M.L has been supported by Ministry of Higher Education and Research, Nord-Pas de Calais Regional Council and FEDER through the Contrat de Projets État-Région (CPER) 2007-2013, as well as by the French National Research Agency (ANR) through the LABEX CEMPI project (ANR-11-LABX-0007). 
References

[1] Hodgkin A. L. and Huxley A. F., The Journal of Physiology, 117 (1952) 500

[2] Channell P., Cymbalyuk G. and Shilnikov A., Phys. Rev. Lett., 98 (2007) 134101.

[3] Channell P., Fuwape I., Neiman A. and Shilnikov A., Journal of Computational Neuroscience, 27 (2009) 527.

[4] Shilnikov A., Nonlinear Dynam., 68 (2012) 305.

[5] BARrio R. and Shilnikov A., Journal of Mathematical Neuroscience, 1 (2011) 6.

[6] Broens M. and BAr-Eli K., The Journal of Physical Chemistry, 95 (1991) 8706.

[7] Wieczorek S., Krauskopf B. and Lenstra D., Phys. Rev. Lett., 88 (2002) 063901.

[8] Ermentrout G. B. and Terman D. H., Mathematical foundations of neuroscience Vol. 35 of Interdisciplinary Applied Mathematics (Springer, New York) 2010.

[9] Korn H. and Faure P., C. R. Biologies, 326 (2003) 787.

[10] Hirata Y., Oku M. and Aihara K., Chaos, 22 (2012) 047511.

[11] Terman D., SIAM J. Appl. Math., 51 (1991) 1418.

[12] Gilmore R., Rev. Modern Phys., 70 (1998) 1455.

[13] Gilmore R., Pei X. and Moss F., Chaos, 9 (1999) 812.

[14] Gilmore R. and Lefranc M., The topology of chaos (Wiley-Interscience [John Wiley \& Sons], New York) 2002.

[15] Channell JR P., Cymbalyuk G. and Shilnikov A., Neurocomputing, 70 (2007) 2107.

[16] Barrio R., Blesa F., Serrano S. and Shilnikov A., Phys. Rev. E, 84 (2011) 035201.

[17] Barrio R., Blesa F. and Serrano S., Physical Review Letters, 108 (2012) 214102.

[18] Letellier C., Dutertre P. and Maheu B., Chaos, 5 (1995) 271.

[19] Barrio R., Blesa F. and Serrano S., Phys. D, 238 (2009) 1087.

[20] Hindmarsh J. L. and Rose R. M., Proc. Roy. Soc. Lond.B, 221 (1984) 87.

[21] Barrio R., Martinez M. A., Serrano S. and Shilnikov A., Chaos, 24 (2014) 023128.

[22] Linaro D., Champneys A., Desroches M. and Storace M., SIAM J. Appl. Dyn. Syst., 11(3) (2012) 939962.

[23] Mindlin G. B., Solari H. G., Natiello M. A., Gilmore R. and Hou X.-J., J. Nonlinear Sci., 1 (1991) 147.

[24] Boulant G., Lefranc M., Bielawski S. and Derozier D., Int. J. Bifurcation Chaos, 8 (1998) 965.

[25] Arai Z., Nonlinear Theory and Its Applications, IEICE, 1 (2013) 104.

[26] Ricca R. and Nipoti B., J. Knot Theory Ramifications, 20 (2011) 1325.

[27] Rosalie M. and Letellier C., J. Phys. A: Math. Theor., 46 (2013) 375101.

[28] Plumecoq J. and Lefranc M., Phys. D, 144 (2000) 231.

[29] Plumecoq J. and Lefranc M., Phys. D, 144 (2000) 259.

[30] Lefranc M., Glorieux P., Papoff F., Molesti F. and Arimondo E., Phys. Rev. Lett., 73 (1994) 1364.
[31] Cvitanovic P., Gunaratne G. and Procaccia I., Phys. Rev. A, 38 (1988) 1503. 